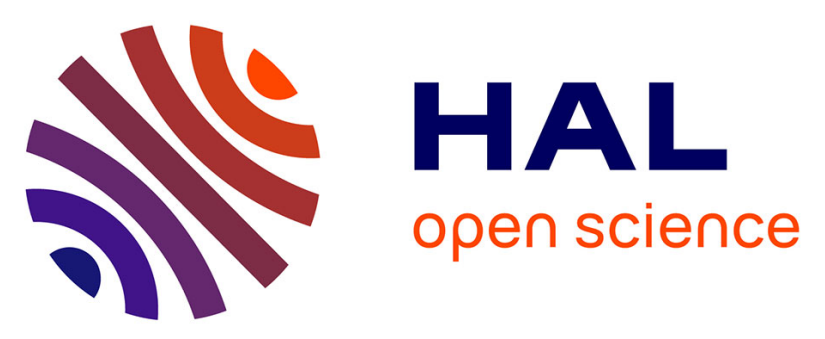

\title{
Numerical Modeling of Discrete Spatial Heterogeneity in Seismic Risk Analysis: Application to Treated Ground Soil Foundation
}

\author{
Silvana Montoya-Noguera, Fernando Lopez-Caballero
}

\section{To cite this version:}

Silvana Montoya-Noguera, Fernando Lopez-Caballero. Numerical Modeling of Discrete Spatial Heterogeneity in Seismic Risk Analysis: Application to Treated Ground Soil Foundation. Georisk: Assessment and Management of Risk for Engineered Systems and Geohazards, 2016, 10 (1), pp.66-82. 10.1080/17499518.2015.1058957 . hal-01163083

\section{HAL Id: hal-01163083 \\ https://hal.science/hal-01163083}

Submitted on 31 Mar 2020

HAL is a multi-disciplinary open access archive for the deposit and dissemination of scientific research documents, whether they are published or not. The documents may come from teaching and research institutions in France or abroad, or from public or private research centers.
L'archive ouverte pluridisciplinaire HAL, est destinée au dépôt et à la diffusion de documents scientifiques de niveau recherche, publiés ou non, émanant des établissements d'enseignement et de recherche français ou étrangers, des laboratoires publics ou privés. 
Georisk

Vol. 00, No. 00, Month 2010, 1-38

NUMERICAL MODELING OF DISCRETE SPATIAL

HETEROGENEITY IN SEISMIC RISK ANALYSIS:

APPLICATION TO TREATED GROUND SOIL FOUNDATION

\author{
S. Montoya-Noguera ${ }^{*}$ and F. Lopez-Caballero ${ }^{a}$ \\ ${ }^{a}$ MSSMat Laboratory CNRS UMR 8579, CentraleSupélec, France \\ (Received 00 Month 20xx; final version received 00 Month 20xx)
}

A finite element soil-structure interaction model is coupled with a discrete autoregressive code, in order to analyze the effect of added spatial variability due to soil improvement in seismic risk analysis. The success of soil improvement techniques is related to the effectiveness of the method - i.e. how much of the soil is being changed - but also to its efficiency in improving the soil behavior i.e. how much are the liquefaction and liquefaction-induced settlement reduced. As these techniques can add spatial variability to a deposit, it can affect the triggering of liquefaction on the soil and the behavior of the structures above it. In this study, this heterogeneity is modeled as a binary mixture, composed by the original liquefiable sand and the added treated sand. The soil behavior is represented by a fully nonlinear elastoplastic multi-mechanism model. The

\footnotetext{
ISSN: $1749-9518$ print/ISSN 1749-9526 online

(C) 2010 Taylor \& Francis

DOI: $10.1080 / 17499518 . Y Y Y Y . x x x x x x$

http://www.informaworld.com
}

*Corresponding author. Email: silvana.montoya-noguera@ecp.fr 
co-seismic settlements of the structure and the liquefaction of the soil deposit are estimated for different effectiveness levels - measured by mixture fractions and for different spatial distributions. In general, both very small or very high mixture fractions presented low efficiency as the improvement in the relative settlement was small. Additionally, results show that the interaction between loose and dense deposits is highly dependent on the spatial distribution. Therefore, homogeneous equivalent models will rarely correspond to the average of the heterogeneous response.

Keywords: Spatial Variability, Seismic Liquefaction, Binary Random Fields

\section{Introduction}

The quantification and consequences of spatial variability and uncertainty in the soil properties is of great importance for geotechnical earthquake engineering. Natural processes and geological formations cause inherent spatial variability; in addition, limited observations and measurement errors add an important amount of uncertainty. Commonly, these aleatory and epistemic uncertainties in a soil deposit are addressed with probabilistic analyses. In those analyses, the soil properties change randomly following a specified probabilistic distribution; although, spatial variability caused by different geological mechanisms is more likely to be a distribution of different soil deposits with horizontal and vertical correlations than a random continuous change (Jones et al. 2002).

While the presence of inherent spatial variability is generally accepted (and addressed by experimental and numerical investigations), the effect of added spatial variability resulting from stabilizing soft soils has been less studied. Methods such as soil-mixing, jet-grouting, bio-grouting and densification techniques are becoming widely used to 
strengthen soft soils and mitigate liquefaction; although, there remains some uncertainties given by the spatial variability introduced in the design (Kasama et al. 2012). One of the most influential factors on the treated ground is the effectiveness of the mixing process (i.e. spatial fraction of the treated soil) which adds important variations with different spatial configurations on the vertical as well as in the horizontal direction (DeJong et al. 2013).

These spatial configurations will have an important impact in complex phenomena like liquefaction, as it depends on the increase of excess pore water pressure $\left(\Delta p_{w}\right)$ and consequent loss of strength. Laboratory tests in heterogeneous soils have shown that the $\Delta p_{w}$ is generated first in loose deposits and when water migrates to neighboring dense sand, it is softened and eventually liquefied. This interaction also affects the liquefactioninduced settlements (Coelho et al. 2004, Dashti et al. 2010, Chakrabortty and Popescu 2012). Extensive research has been performed on the calculation of these settlements but rarely they include the variability of soil properties and modeling of different soil deposits (Koutsourelakis et al. 2002, among others).

The aim of this work is to assess the effect of added spatial variability on the triggering of liquefaction and its consequences. On that account, a 2D finite element model of an inelastic structure founded on loose-to-medium sand is coupled with a discrete auto-regressive model. The latter is used to generate the treated ground soil as a binary mixture composed of two materials: a medium-to-dense sand (MDS) and the remaining loose-to-medium sand (LMS). To assess the efficiency of the mixing process, the co-seismic settlements of the structure founded on a rigid shallow foundation and the liquefaction of the soil deposit are estimated for different levels of effectiveness by ranging the spatial fraction from untreated to fully-treated, with different spatial distributions for each case. 


\section{Previous studies on soil heterogeneity}

As previously stated, two types of spatial variability can be distinguished: (1) inherent spatial variability of soil deposits and (2) spatial variability introduced on the soil by means of increasing its strength and mitigate liquefaction potential.

\section{$2.1 \quad$ Inherent spatial variability}

This type of variability accounts for the dispersion in measured properties in laboratory or in-situ tests, as for the uncertainties in the deposits that have not been measured and the possible errors in the measured quantities or the correlations with mechanical properties (Jones et al. 2002). One way to analyze the effects of spatial variability on liquefaction resistance is through laboratory tests. Undrained cyclic triaxial tests on sand-gravel (Budiman et al. 1995) and sand-silt (Konrad and Dubeau 2002) mixtures, and centrifuge tests on mixtures of sand with different density (Ghosh and Madabhushi 2003, Chakrabortty and Popescu 2012) and with different permeability (Maharjan and Takahashi 2012) evidence the decrease of liquefaction resistance of the mixture compared to that of uniform samples. In general, it was found that the effect of the loose sand zone was to induce increased excess pore water pressure $\left(\Delta p_{w}\right)$ in the surrounding dense sand, or create drainage paths, through which the $\Delta p_{w}$ can be drained out causing differential settlements. While the effect on liquefaction is well evidenced, the effect on the inducedsettlements is yet to be clarified.

Another common approach to account for soil variability and uncertainty is the probabilistic analysis. Popescu et al. (1998) and Fenton and Vanmarcke (1998), among others, conducted several numerical studies on the effects of soil heterogeneity on liquefaction resistance using Monte Carlo (MC) simulations. Their main conclusion on the importance 
of spatial variability in the liquefaction phenomenon, was the presence of pockets that increase the vulnerability of the entire soil deposit and its implications on risk evaluation. Koutsourelakis et al. (2002) and Popescu et al. (2005) presented their results as fragility curves for different parameters as average liquefaction ratio, maximum horizontal displacement, maximum ground settlement and structure tilting. These curves are strongly dependent on the soil properties and seismic motions; therefore, they are only to be used for a qualitative assessment of similar cases.

\section{$2.2 \quad$ Added spatial variability}

Concerning liquefaction mitigation methods, also experimental and numerical analyses can be found. For the former, Hausler and Sitar (2002), Coelho et al. (2004) and Dashti et al. (2010) performed various centrifuge tests to study the densification of liquefiable soils. Important conclusions were drawn with respect to the soil-structure interaction (SSI) effect on the pore-pressure induced softening. Even if a decrease on settlement is evidenced, there appears to be an increase of energy transmitted to the structure. Furthermore, the presence of the structure reduces vertical flow and causes long-term horizontal pore pressure migration from underneath which induces important relative settlements.

Numerically, Bradley et al. (2013) studied soil improvement by jet grouting on the seismic response of a liquefiable deposit. The improved soil was a cementitious material thus no water migration was allowed. Again, all cases were effective at mitigating liquefaction; yet, some presented negative consequences on surface (e.g. large lateral displacements and vertical settlements). Additionally, soil improvement caused an increase in surface acceleration, specially at moderate-to-long periods. The analysis of a structure vulnerability after a liquefaction countermeasure was performed by Lopez-Caballero and 
Modaressi-Farahmand-Razavi (2013). The mitigation method modeled was preloading, and the structural behavior was analyzed by two parameters: relative settlement and the inter-story drift. While the former is reduced after preloading, the latter is increased due to the higher surface amplification. These cases highlight the need for an optimization through which liquefaction resistance could be increased without the decrease in the structure vulnerability.

These examples are important; although, densification by compaction, grouting or mixing produces rarely a homogeneous soil. On the contrary, it results in a heterogeneous mixture of the original liquefiable sand and the added non-liquefiable soil (DeJong et al. 2013). Even if in reality, grouted columns are designed to have specific diameters and spacings, the material is sometimes an heterogeneous mixture of the added soil (or cement) and the original soil. Lambert et al. (2012) performed laboratory tests in samples from soil-cement mixed columns and found heterogeneities in the sides as well as in the core of the columns that consequently affected the mechanical properties. Other improvement processes such as vibro-compaction or bio-grouting have additionally an uncertainty of up to which depth or size the soil is affected, e.g. some areas can be more compacted than others or can have more bacteria-induced cementation. Examples on the latter were shown by in-situ measurements presented by Paassen (2011).

To the best knowledge of authors, there is no numerical or experimental studies related to two-soil mixtures of a liquefiable deposit accounting for both soil and seismic motion variability. These heterogeneous deposits exhibit large, sudden variations from one location to another, unlike natural soil deposits where those variations could be gradual. Therefore, the results of this study may overemphasize the effects of soil heterogeneity on water migration in spatially variable soils. However, as most laboratory soil testing procedures use uniform soil samples for assessing the liquefaction potential, their results 
in terms of cyclic induced $\Delta p_{w}$ may well be on the under-conservative side when applied to natural soil deposits exhibiting inherent or added spatial variability (Chakrabortty and Popescu 2012).

\section{NUMERICAL MODEL}

The numerical model used in this work consists of a reinforced concrete building with a shallow rigid foundation, standing on saturated cohesionless soil. An illustration of the model is shown in figure 1 . The reference soil deposit is a $50 \mathrm{~m}$ width model composed of $20 \mathrm{~m}$ of loose-to-medium (LMS) sand overlaying an elastic bedrock. The shear modulus increases with depth. The fundamental elastic period of the soil profile, calculated with a low-strain frequency analysis, is equal to $0.38 \mathrm{~s}$. It is obtained from the transfer function at free field, i.e. the ratio of the frequency response at the soil surface over the bedrock frequency response for a seismic signal at a very low amplitude to ensure elastic behavior. An elastoplastic multi-mechanism model is used to represent the soil behavior. Under the deposit, an engineering bedrock representing a half-space medium is modeled with an isotropic linear elastic behavior and a shear wave velocity $\left(V_{s}\right)$ equal to $550 \mathrm{~m} / \mathrm{s}$. The ground water table is located $1 \mathrm{~m}$ below the surface.

Concerning the treated ground soil, the recommendations of Mitchell et al. (1998) were used. By which, depth $(d)$ should be given by the extension of the liquefiable layer, in this case $d=4 \mathrm{~m}$ below the water table, and width $(w)$ should extend from the edge of the foundation of size $B$ a distance bigger to the depth of treatment (i.e. $w>B+2 d$ ). The soil heterogeneity is simulated with a discrete auto-regressive-model composed of two materials: a treated medium-to-dense sand (MDS) and the original loose-to-medium sand (LMS). A detailed description of the auto-regressive model is given in section 3.4. 


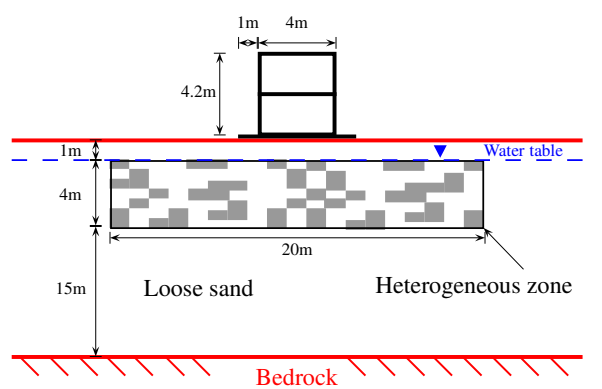

Figure 1.: Illustration of the numerical model

\subsection{Finite Element Model}

A 2D dynamic approach derived from the $\underline{u}-p_{w}$ version of the Biot's generalized consolidation theory (Zienkiewicz and Taylor 1991) was adopted for the soil. The general purpose finite element code GEFDyn (Aubry and Modaressi 1996) was used. The model uses quadrilateral isoparametric elements with eight nodes for both solid displacements and fluid pressures. The thickness of the elements is $0.5 \mathrm{~m}$ and the width is $1 \mathrm{~m}$ under the heterogeneous zone and $1.5 \mathrm{~m}$ on the sides. An implicit Newmark numerical integration scheme is used in the dynamic analysis for the discretization in time. In order to allow an optimal high-frequency dissipation with minimal low-frequency impact, the scheme parameters $\gamma_{N}$ and $\beta_{N}$, that control the precision and stability of the algorithm, are related to the spectral radius at infinity $\left(\rho_{\infty}\right)$. A parametric analysis presented by Montoya-Noguera and Lopez-Caballero (2014) evaluates the induced numerical damping $\left(\xi_{N}\right)$ as a function of this parameter. For this case, $\rho_{\infty}$ was taken equal to 0.8 , thus $\gamma_{N}=0.611$ and $\beta_{N}=0.301$, which correspond to $\xi_{N}=0.1 \%$. It is worth mentioning that this numerical damping affects principally the elastic response of the model (i.e. for shear strains less than $1 \cdot 10^{-5}$ ), while for higher strains the damping is provided by the material degradation as will be shown in section 4 . To take into account the interaction effects between the structure and the soil, a modified width plane-strain condition was assumed in the finite element model (Saez et al. 2013). In this case a width of $4 \mathrm{~m}$ is used 
for the soil deposit.

\subsubsection{Boundary conditions}

The boundary conditions of the finite element model depend on the hypothesis of the analysis. In this case, the medium is considered as an infinite semi-space, the wave propagation is only one-dimensional and purely vertical. Therefore only incident SH waves, i.e. in-plane shear waves in the horizontal direction, propagating in the vertical direction are used as loading. As the response of an infinite semi-space is modeled, equivalent - or periodic - boundaries have been imposed on the lateral nodes. Also referred as tied-nodes, they consist in linking the nodes at opposite sides at the same depth to equal normal stress and equal displacements in all directions. The heterogeneous zone is sufficiently far from the lateral boundaries and so the free field response with and without the treated soil were proved to have the same initial dynamic behavior. For the bedrock's boundary condition, paraxial elements simulating "deformable unbounded elastic bedrock" have been used (Modaressi and Benzenati 1994). The vertically incident shear waves, defined at the outcropping bedrock, are introduced into the base of the model after deconvolution. Thus, the obtained movement at the bedrock interface is composed of both the incident and the reflected waves.

\subsection{Input earthquake motion}

In this paper, focus will be given to the spatial distribution effect on the liquefaction evolution; though, only one motion will be presented. However, the use of other input motions changes the behavior, and this will be treated in a further work. The motion used is the $M_{w}$ 6.7, January 1994, Northridge earthquake recorded in Pacoima Dam in southern California. The unscaled record was taken from the Pacific Earthquake Engineering 


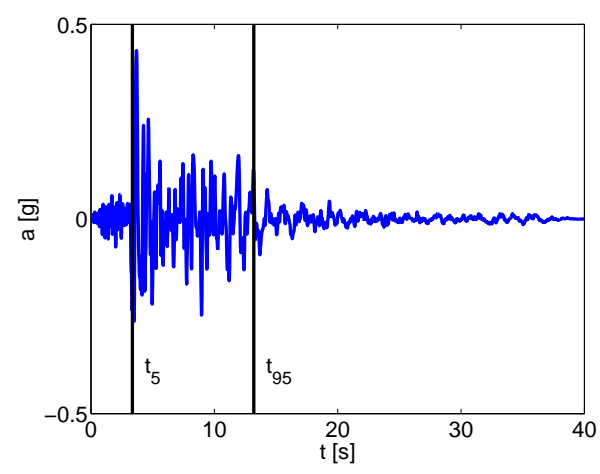

(a)

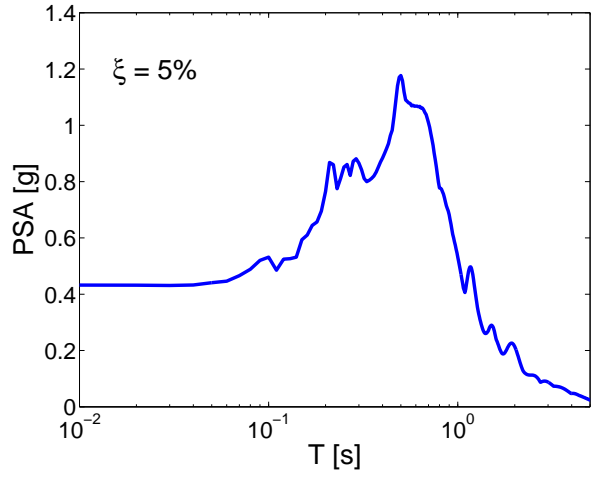

(b)

Figure 2.: Input motion acceleration a) time history and b) response spectra

Research Center (PEER) database under the ID 1050. This station has a site-to-source distance $\left(R_{J B}\right)$ of $4.9 \mathrm{~km}$ and a $30 \mathrm{~m}$-averaged shear-wave velocity $\left(V_{s 30}\right)$ of $2000 \mathrm{~m} / \mathrm{s}$. The peak horizontal acceleration at outcropping (PHA) is $0.43 \mathrm{~g}$, the peak ground velocity $(\mathrm{PGV})$ is $51 \mathrm{~cm} / \mathrm{s}$ and the Arias intensity $\left(I_{A}\right)$ is $1.79 \mathrm{~m} / \mathrm{s}$. Figure 2a shows the acceleration time history, and figure $2 \mathrm{~b}$ the acceleration response spectra with a structural damping $(\xi)$ of $5 \%$. In figure 2 a, the black lines show the time instants for the 5 and $95 \%$ of the Arias intensity. They define the predominant duration $\left(D_{5-95}\right)$, in this case equal to 10 s, where the $90 \%$ of the motion's energy is concentrated. This motion has been widely studied by many authors (Beresnev and Wen 1996, Bonilla et al. 2011, among others) as it is a large event, recorded by many near-to-source permanent strong-motion stations in dense soil conditions.

\section{$3.3 \quad$ Structural model}

For the sake of simplicity a two-story reinforced concrete building proposed by Vechio and Emara (1992) is used. It consists of a large-scale one-span model. The total structure height is $4.2 \mathrm{~m}$ and the width is $4.0 \mathrm{~m}$. The mass of the building is equal to $45 \mathrm{~T}$ and is assumed to be uniformly distributed along beam elements, while the columns are sup- 


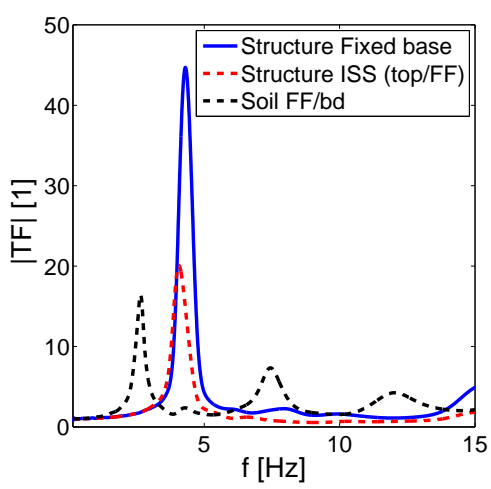

Figure 3.: Transfer function of the structure with fixed base and with SSI effect (top/FF) and of the soil deposit at free-field (surface/bedrock)

posed massless. The fundamental period $\left(T_{s t r}\right)$ is equal to $0.24 \mathrm{~s}$. In order to simulate the structure, plastic hinge beam-column elements are used which take into account axial force $(P)$ and bending moment $(M)$ interaction (Prakash et al. 1993). The foundation is modeled as a rigid block of $0.1 \times 6 \times 4 \mathrm{~m}$. It is based on the two-component model presented by Giberson (1969) and the modifications introduced by Prakash et al. (1993) to take into account axial force $(P)$ and bending moment $(M)$ interaction by specifying $P-M$ yield surfaces. Figure 3 shows the transfer function (TF) of the free-field (surface/bedrock) and of the structure at fixed base and with soil-structure interaction (SSI) effects (top/FF). The transfer function is the ratio between two acceleration wave fields and it gives information solely of the soil between these two points. The SSI effects result on a shift of the main frequency of the structure due to the flexibility of the foundation soil and a deamplification due to the material and radiation damping added by the soil.

\subsection{Spatial discretization}

The models to generate binary random fields can be divided in two main approaches according to their definition of spatial dependence: the Gaussian Markov random field (GMRF) models that treats dependence indirectly (Banerjee et al., 2004) and the autologistic models that does it directly through the so-called autocovariate, i.e. a function 
of the observations themselves. The homogeneous auto-logistic model was derived by Bartlett and Besag (1969) and is a nearest-neighbor model, defined as a conditional probability; it is intuitive and appealing. On the contrary, the GMRF models, firstly introduced by Whittle (1963), are defined as a joint probability distribution. The spatial discretization for this work follows the auto-logistic model. It is important to remark that the validation of these models are out of the scope of this paper.

The binary mixture used to model the heterogeneous zone is defined with the spatial fraction $\gamma=N_{1} /\left(N_{1}+N_{2}\right)$ where $N_{m}$ is the number of elements of material $m$. From an engineering point of view, $\gamma$ is related, for example, to the efficiency of a soil improvement technique, such as soil-mixing. Thus, $\gamma$ could be calculated from the injected material with respect to the total area of intervention. The model is discretized into a rectangular grid, where each element is assigned one of the possible values: 0 (material 1) or 1 (material 2). The expectation of $x_{i j}$, a value of the binary random variable $X_{i j}$, is given by:

$$
E\left[x_{i j} \mid x_{i-1, j}, x_{i, j-1}\right]=\left[1-\frac{1}{2}\left(\beta_{1}+\beta_{2}\right)\right] \cdot \gamma+\frac{1}{2}\left(\beta_{1} \cdot x_{i-1, j}+\beta_{2} \cdot x_{i, j-1}\right)
$$

where $\gamma$ is the spatial fraction and $\beta_{1}$ and $\beta_{2}$ are the auto-regressive coefficients that give the one step correlation of the process in each direction. Which means that the element expectation $\left(E\left[x_{i j}\right]\right)$ is influenced by its nearest-neighbors $x_{i-1, j}$ and $x_{i, j-1}$. Under the condition of homogeneity, the $E\left[x_{i j}\right]$ is independent of the internal position $(i, j)$ on the array. For each element, the generated probability is not a binary number, so it is compared to a random number $\left(u_{i j}\right)$ that follows a uniform distribution function between 0 and 1 , where each element is independent. This process, known as binarization, makes use of MC simulations to converge to a given $\gamma$ value. 
The procedure to generate the field of size $m \times n$ is schematized in figure 4 and has the following steps:

(1) Assign the value of the corner element $\left(x_{i=1, j=1}\right):$ As it is assumed that the model tends to homogeneity, $E\left[x_{1,1}\right]$ equals $\gamma$, thus the binarization process turns to:

$$
x_{1,1}= \begin{cases}0, & \text { if } \gamma \leq u_{1,1}[0,1] \\ 1, & \text { otherwise }\end{cases}
$$

(2) Assign the values for the lower horizontal boundary $x_{i=2, \ldots, m, 1, j=1}$ : Based on the 1D model where $\beta_{1}=\beta_{2}$, so:

$$
E\left[x_{i, 1} \mid x_{i-1,1}\right]=\left(1-\beta_{1}\right) \gamma+\beta_{1} \cdot x_{i-1,1}
$$

Again if $E\left(x_{i, 1}\right) \leq u_{i, 1}$, then $x(i, 1)=0$; otherwise, $x(i, 1)=1$.

(3) Repeat previous step but for the left vertical boundary $x_{i=1, j=2, \ldots, n}$ :

$$
E\left[x_{1, j} \mid x_{1, j-1}\right]=\left(1-\beta_{2}\right) \gamma+\beta_{2} \cdot x_{1, j-1}
$$

(4) Fill up the interior of the rectangle $x_{i=2, \ldots, m, j=2, \ldots, n}$ : 2D model with equation 1.

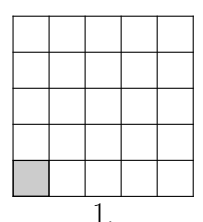

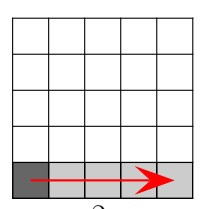

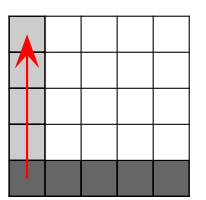

3.

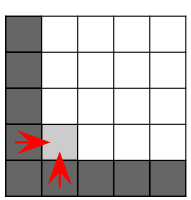

4.

Figure 4.: Procedure scheme for the auto-regressive discretization model

As an example, a zoom on the heterogeneous zone of two spatial distributions are shown 
in figure 5 and correspond to a spatial fraction $(\gamma)$ of 0.4 and auto-regressive coefficients $\left(\beta_{1}\right.$ and $\beta_{2}$ ) equal to 0.4 . As it can be seen, $\beta$ does not give a constant correlation length in each row or column, but it is an average on the model. Similarly, $\gamma$ is the average over the entire area; even though, there are regions with different composition as in the top left corner in figure 5a.

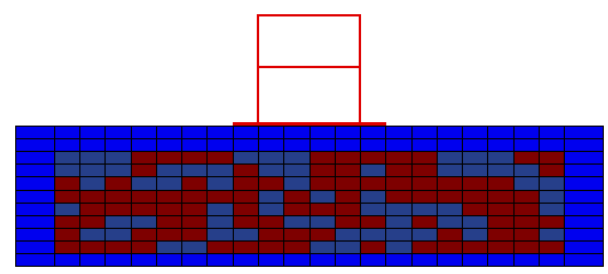

(a)

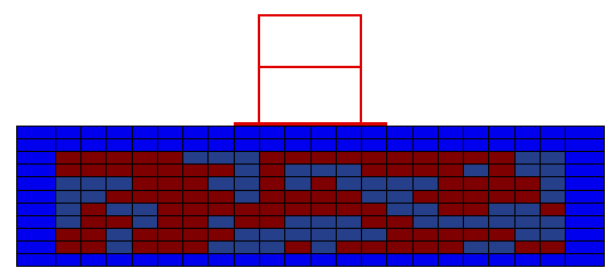

(b)

Figure 5.: Zoom on the heterogeneous zone for 2 distributions with $\gamma=0.4$ and $\beta_{1}=\beta_{2}$ $=0.4$

\section{Soil constitutive model}

The soil behavior is simulated with the ECP elasto-plastic multi-mechanism model, which is an advanced step-by-step integration model developed at Ecole Centrale Paris (Aubry et al. 1982, Hujeux 1985). The model is written in terms of effective stresses, uses a Coulomb type failure criterion and follows the critical state concept. The evolution of hardening is based on the plastic strain (deviatoric and volumetric strains for the deviatoric mechanisms and only volumetric strains for the isotropic one). To take into account the cyclic behavior it uses a kinematical hardening which relies on the state variables, at the last load reversal. The soil behavior is decomposed into pseudo-elastic, hysteretic and mobilized domains hence it can take into account a large range of deformations. A brief description of the ECP model will be given below; however, for a complete understanding refer to Aubry et al. (1982) and Hujeux (1985), among others. 
The pseudo-elastic domain is isotropic and nonlinear, where the bulk $(K)$ and the shear $(G)$ moduli increase with the mean effective stress $\left(p^{\prime}\right)$, as follows: $K_{\text {max }}=K_{r e f}\left(p^{\prime} / p_{r e f}^{\prime}\right)^{n_{e}}$ and $G_{\max }=G_{r e f}\left(p^{\prime} / p_{r e f}^{\prime}\right)^{n_{e}}$, where $K_{r e f}$ and $G_{r e f}$ are the moduli at the reference stress $\left(p_{r e f}^{\prime}\right)$ and $n_{e}$ is the degree of nonlinearity. The model takes into account four mechanisms: three deviatoric and one isotropic. Adopting the soil mechanics sign convention (i.e. compression positive), the deviatoric primary yield surface on the $k$ plane is:

$$
f_{k}\left(\underline{\underline{\sigma^{\prime}}}, \varepsilon_{v}^{p}, r_{k}\right)=q_{k}-\sin \phi_{p p}^{\prime} \cdot p_{k}^{\prime} \cdot F_{k} \cdot r_{k}
$$

where $p_{k}^{\prime}$ and $q_{k}$ are the mean and deviatoric values of the stress tensor, and $\phi_{p p}^{\prime}$ is the friction angle at the critical state. The isotropic hardening associated with the volumetric strain is controlled by:

$$
F_{k}=1-b \ln \left(\frac{p_{k}^{\prime}}{p_{c}}\right)
$$

in which, the parameter $b$ gives the form of the yield surface in the $p^{\prime}-q$ plane and varies from 0 (Coulomb type) to 1 (Cam-Clay). $p_{c}$ is the mean stress at the critical state and is defined as $p_{c}=p_{c 0} \exp \left(\beta \varepsilon_{v}^{p}\right)$ with $p_{c 0}$ being the stress corresponding to the critical state of the initial density, and $\beta$, the plastic compressibility modulus. Lastly, the isotropic hardening generated by plastic shearing is controlled by the degree of mobilized friction:

$$
r_{k}=r_{k}^{e l a}+\left(\frac{\bar{\varepsilon}_{k}^{p}}{a+\bar{\varepsilon}_{k}^{p}}\right)
$$

that varies from the $r_{k}^{e l a}$, i.e. the size of the elastic deviatoric domain, to the unit value.

The ECP model has been validated for different kinds of loading and the main results are shown in many publications; among others, Costa D'Aguiar et al. (2011), Saez et al. (2013) for reproducing laboratory tests, Foerster and Modaressi (2007), Bernardie et al. (2006) for seismic soil response of vertical arrays, and Sica et al. (2008) for seismic 
response of soil structures.

\subsection{Laboratory tests simulations}

The soils used in the numerical model are two sands that present different relative densities: LMS, loose-to-medium and MDS, medium-to-dense. Their model parameters were calibrated using the procedure defined by Lopez-Caballero et al. (2007) and validated with laboratory tests by Lopez-Caballero and Modaressi-Farahmand-Razavi (2013) and Saez (2009), respectively. The parameters are divided into five groups corresponding to the different mechanisms, a description and the values used are shown in table 1. Additionally, the parameters of the soil used in the deeper layers, also taken from LopezCaballero and Modaressi-Farahmand-Razavi (2013), are given in the same table. It should be noted that the ECP model regards the initial relative densities as consequence of some intrinsic soil parameters, e.g. the soil density $\left(\rho_{s}\right)$ and the porosity $\left(n_{0}\right)$, the initial state given by the critical stress $\left(p_{c 0}\right)$ and the void ratio $(e)$. The hydraulic conductivity $(K)$ is different for the two soils and was given by the previously cited authors. For a better understanding of the soils behavior, three laboratory tests were simulated.

Drained cyclic shear test are simulated for both soils. The confining pressures $\left(p_{0}\right)$ under which the soil was tested were 50,100 and $150 \mathrm{kPa}$. The tests results for the MDS are shown in figure 6 . The other soil has similar curves and are not shown here for the sake of brevity. The obtained shear degradation curves $\left(G / G_{\max }-\gamma\right)$ match relatively well with the reference given by Seed et al. (1986); whereas for the damping (D) it can be observed an underestimation for strains less than $0.01 \%$ and an overestimation for large strains. The underestimation for low strains, is due to the elasto-plastic model implementation and is overcome by the addition of numerical damping (see section 3.1). 


\begin{tabular}{|c|c|c|c|c|c|}
\hline Parameter description & LMS & MDS & {$[5-10] \mathrm{m}$} & {$[10-15] \mathrm{m}$} & [15-20]m \\
\hline$\overline{\rho_{s}}\left[\mathrm{~kg} / \mathrm{m}^{3}\right]$ : Solid density & 2700 & 2700 & 2700 & 2700 & 2700 \\
\hline$n_{0}:$ Initial porosity & 0.47 & 0.35 & 0.47 & 0.47 & 0.47 \\
\hline$k_{0}$ : Coefficient of earth pressure & 0.6 & 0.6 & 0.6 & 0.6 & 0.6 \\
\hline$K[\mathrm{~m} / \mathrm{s}]:$ Hydraulic conductivity & $1 \cdot 10^{-4}$ & $1 \cdot 10^{-5}$ & $1 \cdot 10^{-4}$ & $1 \cdot 10^{-4}$ & $1 \cdot 10^{-4}$ \\
\hline \multicolumn{6}{|l|}{ Elasticity } \\
\hline$K_{\text {ref }}[M P a]:$ Bulk modulus & 628.0 & 444.0 & 628.0 & 628.0 & 628.0 \\
\hline$G_{r e f}[M P a]:$ Shear modulus & 290.0 & 222.4 & 290.0 & 290.0 & 290.0 \\
\hline$n_{e}:$ Nonlinear degree & 0.5 & 0.4 & 0.5 & 0.5 & 0.5 \\
\hline$p_{r e f}[M P a]:$ Reference mean stress & 1.0 & 1.0 & 1.0 & 1.0 & 1.0 \\
\hline \multicolumn{6}{|l|}{ Critical State and Plasticity } \\
\hline$\phi_{p p}^{\prime}\left[{ }^{\circ}\right]:$ Friction angle & 30 & 31 & 30 & 30 & 30 \\
\hline$\beta$ : Plastic compressibility modulus & 33 & 43 & 33 & 33 & 33 \\
\hline$d$ : Isotropic consolidation distance & 2.00 & 3.50 & 2.00 & 2.00 & 2.00 \\
\hline$b:$ Yield surface shape & 0.20 & 0.20 & 0.20 & 0.20 & 0.20 \\
\hline$p_{c o}[M P a]$ : Initial critical stress & 0.019 & 1.800 & 0.040 & 0.061 & 0.082 \\
\hline \multicolumn{6}{|l|}{ Flow Rule and Isotropic Hardening } \\
\hline$\psi\left[^{\circ}\right]:$ Characteristic angle & 30 & 31 & 30 & 30 & 30 \\
\hline$\alpha_{\psi}:$ Volumetric parameter & 1.0 & 1.0 & 1.0 & 1.0 & 1.0 \\
\hline$a_{1}:$ Primary plastic stiffness & $1 \cdot 10^{-4}$ & $1 \cdot 10^{-4}$ & $1 \cdot 10^{-4}$ & $1 \cdot 10^{-4}$ & $1 \cdot 10^{-4}$ \\
\hline$a_{2}:$ Secondary plastic stiffness & $2 \cdot 10^{-3}$ & $4 \cdot 10^{-3}$ & $2 \cdot 10^{-3}$ & $2 \cdot 10^{-3}$ & $2 \cdot 10^{-3}$ \\
\hline$c_{1}$ : Monotonic isotropic hardening & $1 \cdot 10^{-3}$ & $3 \cdot 10^{-2}$ & $1 \cdot 10^{-3}$ & $1 \cdot 10^{-3}$ & $1 \cdot 10^{-3}$ \\
\hline$c_{2}:$ Cyclic isotropic hardening & $2 \cdot 10^{-3}$ & $6 \cdot 10^{-2}$ & $2 \cdot 10^{-3}$ & $2 \cdot 10^{-3}$ & $2 \cdot 10^{-3}$ \\
\hline$m$ : Cyclic loading exponential & 1.5 & 1.0 & 1.5 & 1.5 & 1.5 \\
\hline \multicolumn{6}{|l|}{ Threshold Domains } \\
\hline$r^{\text {ela }}:$ Elastic & $3 \cdot 10^{-2}$ & $5 \cdot 10^{-3}$ & $2 \cdot 10^{-2}$ & $1 \cdot 10^{-2}$ & $5 \cdot 10^{-3}$ \\
\hline$r^{\text {hys }}:$ Hysteretic & $4 \cdot 10^{-2}$ & $3 \cdot 10^{-2}$ & $4 \cdot 10^{-2}$ & $4 \cdot 10^{-2}$ & $4 \cdot 10^{-2}$ \\
\hline$r^{m o b}:$ Mobilized & $8 \cdot 10^{-1}$ & $8 \cdot 10^{-1}$ & $8 \cdot 10^{-1}$ & $8 \cdot 10^{-1}$ & $8 \cdot 10^{-1}$ \\
\hline$r_{i s o}^{e l a}:$ Isotropic elastic & $1 \cdot 10^{-3}$ & $1 \cdot 10^{-3}$ & $1 \cdot 10^{-3}$ & $1 \cdot 10^{-3}$ & $1 \cdot 10^{-3}$ \\
\hline
\end{tabular}

Table 1.: ECP model parameters

Furthermore, a drained triaxial test was modeled for different confining pressures. The results of the volumetric strains $\left(\varepsilon_{v}\right)$ as a function of the deviatoric strains $\left(\varepsilon_{1}\right)$ for both soils are presented in figure 7a. For the LMS, the volumetric strains are below zero, which means that the soil deposit is principally contracting. On the contrary, the MDS presents a dilatant behavior for the same initial stress.

Finally, an undrained stress controlled cyclic shear test was simulated. The cyclic stress ratio $\left(S R=\tau / \sigma_{v 0}^{\prime}\right)$ as a function of the number of loading cycles to produce liquefaction $(N)$ is shown in figure $7 \mathrm{~b}$ for both soils. As a qualitative comparison, the modeled test 

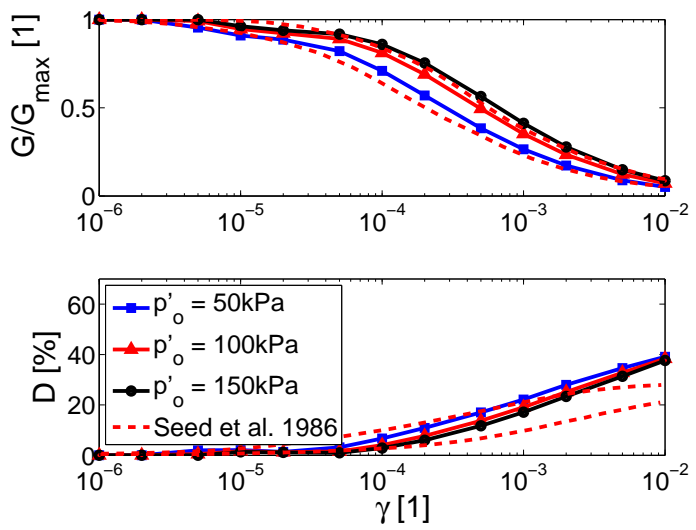

Figure 6.: Simulated a) shear degradation and b) damping curves for the MDS

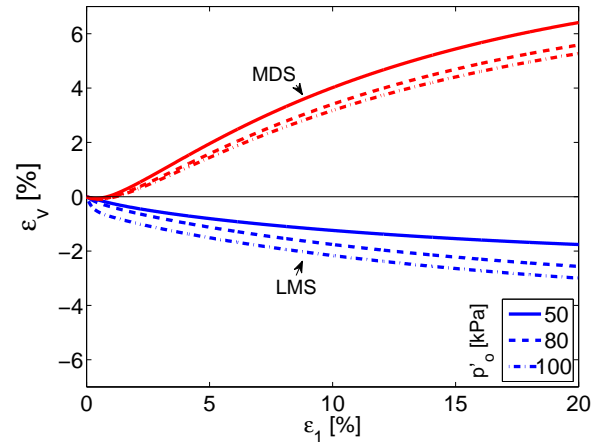

(a)

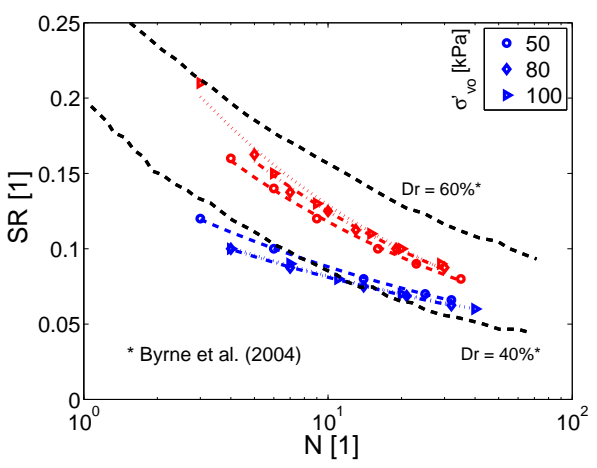

(b)

Figure 7.: Simulated laboratory tests: a) Volumetric strain $\left(\varepsilon_{v}\right)$ as a function of the deviatoric strain $\left(\varepsilon_{1}\right)$ and b) Liquefaction curves for both soils

results are compared with the curves given by Byrne et al. (2004) for Nevada sand at different densities (i.e. $D_{r}=40$ and $60 \%$ ). It is noted that the obtained curves are closer to the reference for a $D_{r}=40 \%$ corresponding to the LMS; while, the MDS curves are closer to those of a $D_{r}=60 \%$.

\section{Liquefaction analysis}

In this section, the response of the model without treatment is analyzed. This is the reference case when $\gamma=1$. Figure 8 a shows the pore pressure ratio $\left(r_{u}=\Delta p_{w} / \sigma_{v 0}^{\prime}\right)$ at the end of the shaking (i.e. co-seismic analysis). When $r_{u}$ is equal to unity, the soil has loosened all its strength and it is totally liquefied. As it can be observed, most part of 


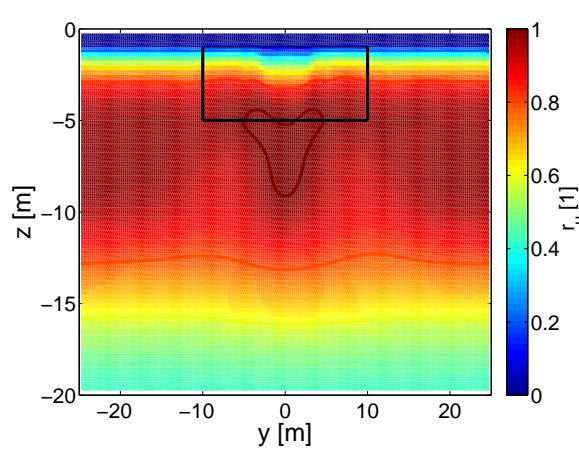

(a)

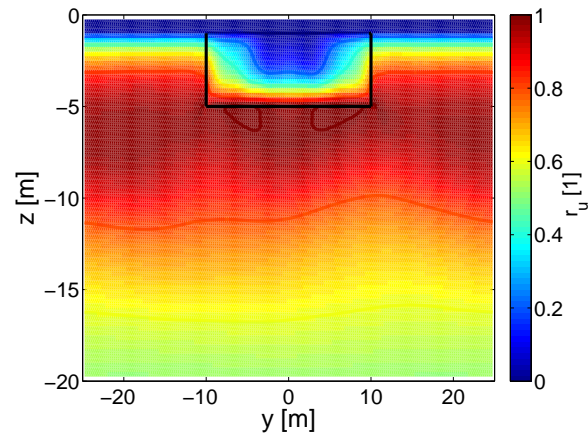

(b)

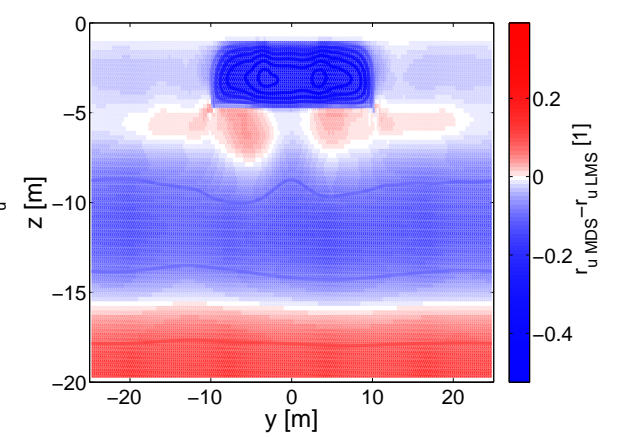

(c)

Figure 8.: Liquefaction ratio $r_{u}$ at the end of shaking: a) before and b) after soil treatment and c) difference between them

the deposit is liquefied, with the highest ratio above $5 \mathrm{~m}$.

The relative settlement of the structure with respect to free-field at the end of shaking $\left(\left|u_{z}\right|\right)$ is $23 \mathrm{~cm}$, which according to the limits given by Bird et al. (2006) corresponds to a moderate damage state. Figure 9 shows the co-seismic and post-seismic relative settlement and liquefaction ratio under the right column and at 3.5m depth. Although, all $\Delta p_{w}$ is not dissipated, there is almost no contribution of post-seismic consolidation to the total settlement (i.e. less than $0.2 \%$ ). By comparing the time histories with the acceleration at outcropping, also shown in figure 9, it is interesting to note that while the high acceleration peak produces the biggest increase in the liquefaction ratio, the relative settlement is gradually increased and its rate is higher after 10s. At this time a second peak in acceleration is evidenced at higher frequencies which is probably also affecting the MDS $\Delta p_{w}$ evolution.

\section{Liquefaction mitigation analysis}

Deep mixing techniques like jet-grouting, bio-grouting or wet-soil mixing use stiffer materials to form foundation elements to stabilize soft soils and mitigate liquefaction. These techniques are widely used even though there remains some uncertainties given the spa- 


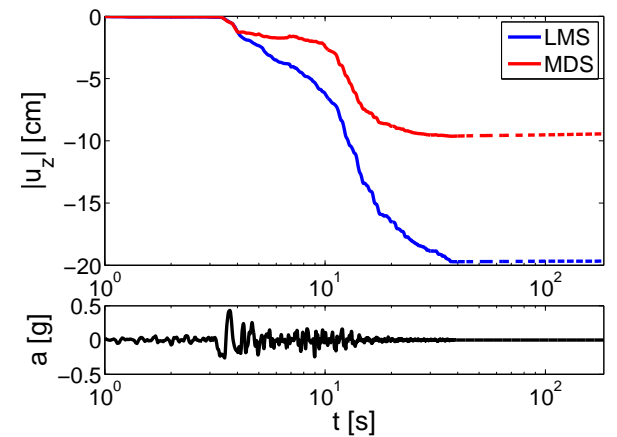

(a)

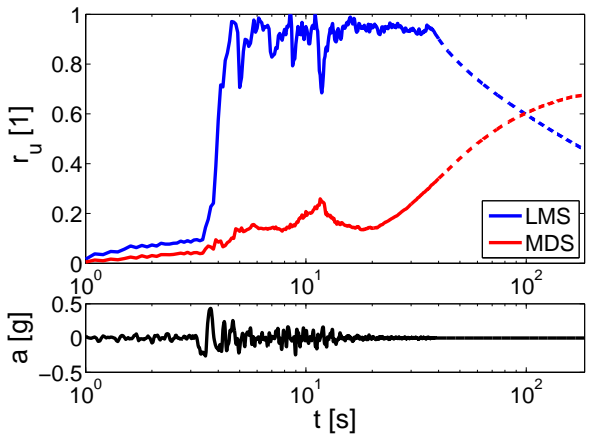

(b)

Figure 9.: Time histories of the (a) Relative settlement $\left|u_{z}\right|$ and the (b) Liquefaction ratio $r_{u}$ under the right column and at $3.5 \mathrm{~m}$ depth before and after mitigation. Post-seismic behavior is depicted with dotted lines. The acceleration time history at outcropping is shown below

tial variability introduced in the design (Kasama et al. 2012). Sometimes, given the poor soil conditions and the high seismic demand, soil mixing is used to replace an entire portion of the deposit. This last case is analyzed and is also used as reference $(\gamma=0)$. The liquefaction ratio at the end of shaking is shown in figure $8 \mathrm{~b}$. It is interesting to note that while $r_{u}$ decreases directly under the structure, it increases on the surrounding soil. Figure $8 c$ shows the difference between the two cases. Negative values, in blue, denote the places where the liquefaction is greater before treatment (LMS case) and the places where liquefaction increases after treatment (MDS case) are the positive values, shown in red. Two zones presented higher $r_{u}$ values after treatment: (1) a layer of a couple of meters immediately below the treatment zone and (2) the soil below $15 \mathrm{~m}$.

In an effort to quantify the excess pore-pressure generation, Shinozuka and Ohtomo (1989) introduced the liquefaction Index $(Q)$ applied in this case to the area of the model as:

$$
Q_{A}=\frac{1}{H \cdot L} \int_{0}^{H} \int_{0}^{L} r_{u}(y, z) \mathrm{d} y \mathrm{~d} z
$$

where $r_{u}$ is the liquefaction ratio evaluated at the end of shaking and $H$ and $L$ are 
the height and length of the soil deposit. A value of 1.0 indicates conditions of initial liquefaction throughout the $H \cdot L$ area and thus gives information of the liquefaction ratio as well as the total liquefied zone. After the entire portion of the soil is replaced, and if $Q_{A}$ is evaluated in the mitigation zone (i.e. $H=4 \mathrm{~m}$ and $L=20 \mathrm{~m}$ ), it decreases $44 \%$. However for the entire soil deposit (i.e. $H=20 \mathrm{~m}$ and $L=50 \mathrm{~m}$ ) the use of soil mixing only decreases $6 \%$ the $Q_{A}$ value. Which means that liquefaction in the model is reduced under the structure, but is present in other locations. These results are in accordance with those of Coelho et al. (2004) and Lopez-Caballero and Modaressi-Farahmand-Razavi (2013) and can be explained by the energy of the motion that must be dissipated. As it is constant for both cases, the reduction in one zone, will necessarily affect other parts of the deposit. Additionally, the pore-pressure migration can be seen from the loose untreated sand to the mitigation zone in the boundaries between both soils. An important interaction is therefore present in the mixture which will be of great importance in the heterogeneous deposit case.

Concerning the relative settlement $\left(\left|u_{z}\right|\right)$, shown in figure 9 a, a reduction of almost $50 \%$ is attained, which according to the limits given by Bird et al. (2006) corresponds to a slight damage state and will only need repairs for aesthetic reasons. As the pore-pressure migration continues after shaking, the liquefaction ratio under the right column and at $2.5 \mathrm{~m}$ depth increases (shown in figure $9 \mathrm{~b}$ ); although, there is almost no contribution of post-seismic consolidation to the total settlement.

\subsection{Heterogeneous model}

One of the most influential factors on the treated ground is the effectiveness of the mixing process, which adds important variations in the soil. This heterogeneity depends on the mitigation technique, but will in general have a spatial correlation on the vertical 
and horizontal direction. Studies on this topic usually apply homogeneous random field techniques to account for the spatial variation (Jones et al. 2002). In them, properties are described by a probabilistic function where a mean value and a coefficient of variation is provided. In the present analysis, it was assumed that the heterogeneous deposit is composed of two materials: a treated-soil and the remaining soft soil. This could be the case of mixing techniques such as soil-mixing or bio-treatment. The interaction of these soils is important as the loose sand induces an increase in excess pore-water pressure in the surrounding treated sand.

As recalled before, Chakrabortty and Popescu (2012) analyzed centrifuge experiments of heterogeneous sand deposits. Results show an increase in excess pore water pressure for the heterogeneous case compared with an average-density homogeneous sand. However, the analysis was deterministic and the use of a traditional homogeneous equivalent model could be inappropriate due to the fully nonlinear behavior. The traditional homogenization theories, such as the linear and harmonic average bounds (Wiener 1912) are used to describe the effective properties of parallel or perpendicular phase alignment but the geometric arrangements of granular soil and interactions when subjected to seismic loading are rarely fitted to these cases. The spatial discretization described in section 3.4 is used here to generate the heterogeneous deposits. The spatial fraction $(\gamma)$ is varied between not treated to fully treated (i.e. from 1 to 0 ) and 20 independent spatial distributions per $\gamma$ value were realized.

The auto-regressive coefficients $\beta_{1}$ and $\beta_{2}$ control the correlation length in the horizontal and vertical direction. These parameters, which are normalized by the size of the heterogeneous zone, describe the degree of correlation of the spatially random values. Thus, a value close to 1 will imply a smoothly varying field, while a small value will imply a ragged field. In this analysis, both coefficients are equal to 0.4 (i.e. a correlation 
length close to $8 \mathrm{~m}$ ). This value was taken from in-situ measurements and recommendations for sandy soil and gravelly sand (Alonso and Krizek 1975, Senger et al. 1992). The effect in the liquefaction-induced settlement of different spatial correlations (e.g. magnitude of the correlation lengths and differences between the horizontal and the vertical correlations) could be interesting but it is out of the scope of this paper.

\subsection{Water migration and soil-interaction in a heterogeneous deposit}

In order to better understand the interaction between the two soils in the heterogeneous deposit, the results for different treatment efficiencies will be analyzed.

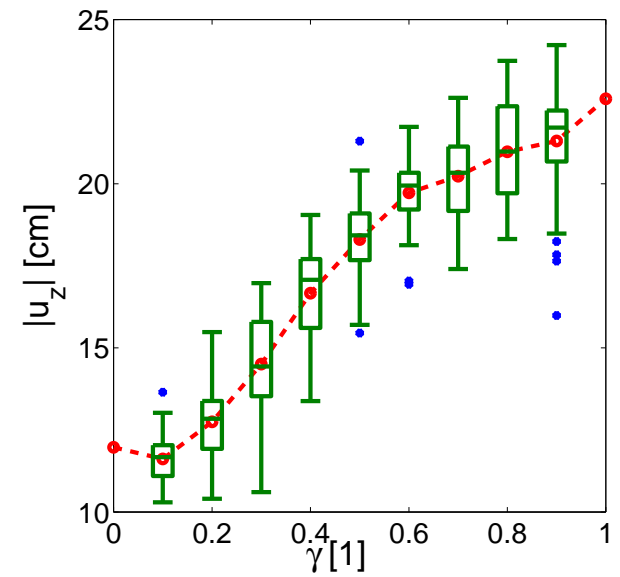

(a)

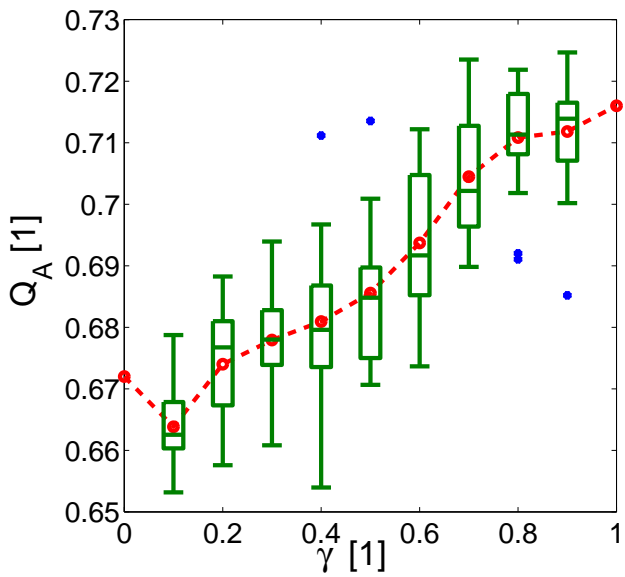

(b)

Figure 10.: Box-and-whiskers plot for a) the relative settlement $\left(\left|u_{z}\right|\right)$ of the structure with respect to free-field and b) the Liquefaction Index $\left(Q_{A}\right)$

Figure 10 shows the box-and-whisker plots for the relative settlement of the structure with respect to free-field $\left(\left|u_{z}\right|\right)$ and for the liquefaction index evaluated at the treated zone $\left(Q_{A}\right)$ as a function of the spatial fraction. These plots are commonly used to show scalar statistics when the uncertainty of a number of related variables are compared (Helton et al. 2006). In fact, they give information not only about the mean values (in red), but also about the 3 quartiles, corresponding to 25,50 and $75 \%$ of data (that compose the box) and the extreme cases, as the whiskers are the lowest and highest data 
within 1.5IQR (Inter-Quartile Range) and the unusual observations or outliers are drawn as blue dots. In order to analyze the effect of the treated soil in the relative settlement, concerning figure 10a, two aspects will be discussed : the variation of the average value and the dispersion for each $\gamma$ value.

At first, the efficiency of the soil improvement can be analyzed from the evolution of the average relative settlement with the spatial fraction, shown in figure 10a in red. For this case, it seems that even a small amount of treated soil (e.g. $\gamma=0.9)$ can reduce, in average, the relative settlement. In contrast, it appears that below a certain spatial fraction, the average settlement will not be greatly affected if a greater amount of denser soil is used. In other words, it seems that the relation between the relative settlement and the spatial fraction is nonlinear and divided in two concave curves: one that is mostly related to the natural soil (higher $\gamma$ values) and another highly sensitive to the introduced soil (lower $\gamma$ values).

Furthermore, regarding all values it can be observed that higher variation is present for $\gamma$ equal to 0.3 . This value could be the percolation threshold dividing the two curves, from which the interactions between the two soils change. It can be noted that this relation differs greatly from the average value of both soils, hence, traditional homogenization theories - such as the arithmetic (parallel) or geometric (series) averages should not be used as reference. Otherwise, while for some spatial fractions the average value will be overestimated; for others, it will be underestimated.

From a statistical point of view, the outliers have an important effect in the probabilistic distribution function (PDF). In other words, the existence of these values can change the PDF for example from a lognormal to a Gumbel distribution. For $\gamma_{L M S}=0.9$, more outliers are seen; which is due to the very different spatial distributions that can be presented. A zoom on the heterogeneous zone of the spatial distributions that presented 
the minimum and maximum $\left|u_{z}\right|$ are shown in figure 11 and correspond to 16.0 and 23.4 $\mathrm{cm}$, respectively. It is noted that for the former, i.e. minimum $\left|u_{z}\right|$ in figure $11 \mathrm{a}$, the MDS elements (colored in red) are on shallow depths, mostly under the structure. Even so a cluster is present under the right column. On the contrary, for the maximum $\left|u_{z}\right|$, in figure 11b, the soils are in smaller clusters and the MDS elements are mostly at the bottom or near the edges. These conclusions agree with the theory of stress distribution of Boussinesq, hence as the inclusions are further from the structures region of influence their effect is smaller.

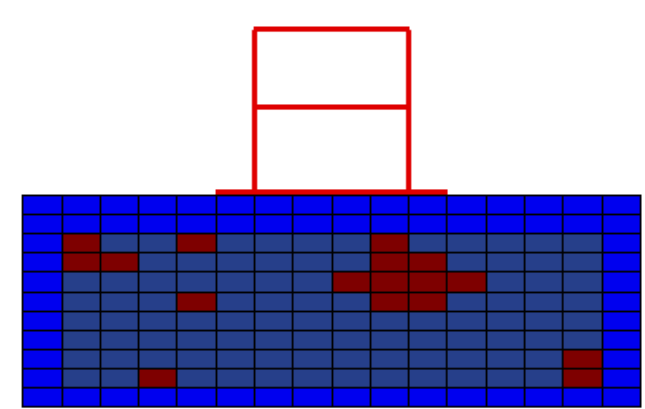

(a)

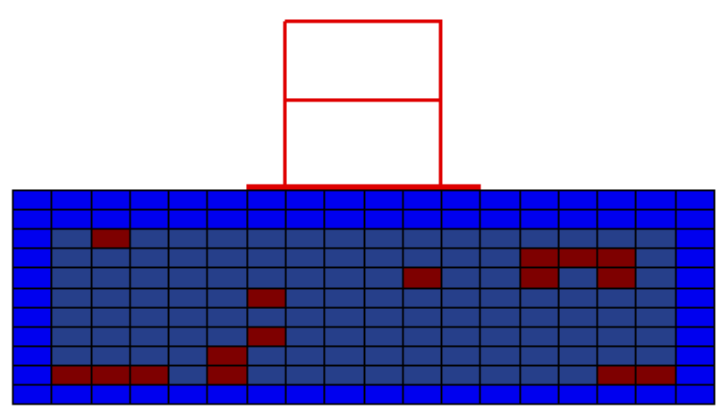

(b)

Figure 11.: Zoom on the heterogeneous zone for the distributions with $\gamma=0.9$ and $\beta_{1}=\beta_{2}$ $=0.4$ that presented a) the minimum and b) maximum $\left|u_{z}\right|$

However, the variation on the box and whiskers size shown in figure 10a, suggests that the discrete model is highly dependent on the interaction between the two materials and their spatial distribution. In this regard, figure 12a shows the experimental cumulative density function (CDF) for 3 cases: $\gamma$ equal to $0.3,0.5$ and 0.7 . The $\left|u_{z}\right|$ is normalized with respect to the corresponding mean value to allow the comparison of the variation. It can be observed that for $\gamma$ equal to 0.3 , the CDF has a lower slope though it presents higher variation.

Regarding figure 10b, there is a clear relationship between the $\left|u_{z}\right|$ and the $Q_{A}$, which explains the importance of pore water pressure generation and migration through the soil 


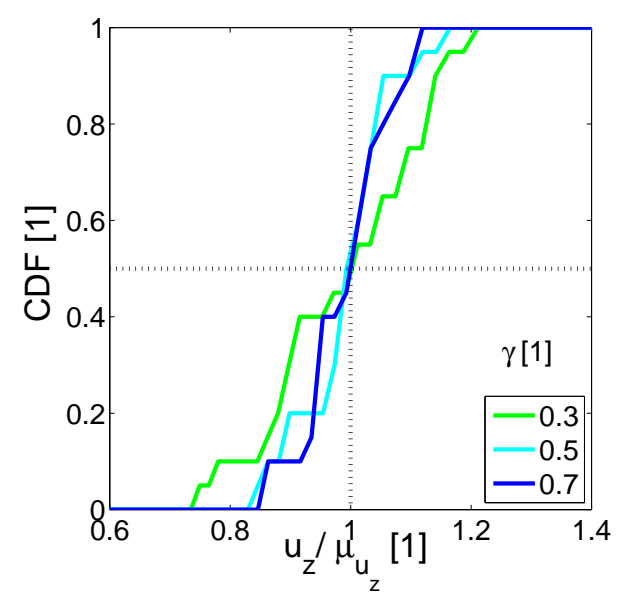

(a)
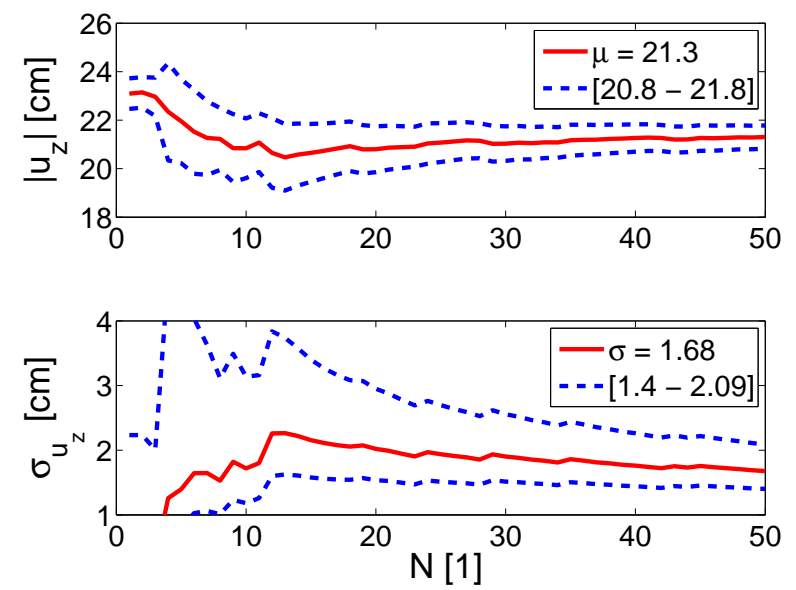

(b)

Figure 12.: Relative settlement $\left(\left|u_{z}\right|\right)$ : a) cumulative density function and b) Convergence of the mean and standard deviation for $\gamma=0.9$ and $\beta_{1}=\beta_{2}=0.4$

deposit. However, the $Q_{A}$ presents higher variation specially for lower spatial fractions. A possible explanation is that liquefaction triggering depends on the spatial distribution and the presence of clusters while the relative settlement depends on the entire deposit. This subject will be explained below.

In order to verify that the number of distributions used is sufficient for the purpose of this work, the statistical convergence of the mean and the standard deviation of the relative structure settlement is shown in figure $12 \mathrm{~b}$ for $\gamma$ equal to 0.9 . The blue dashed lines correspond to the $5 \%$ and $95 \%$ confidence intervals obtained with the t-student and $\chi^{2}$ statistical model, respectively. Although there is still some variation in the results, for $\mathrm{N}=20$ the statistical convergence is satisfactory for this analysis.

However, for the same spatial fraction, the relative settlement can vary greatly only due to the distribution of the two soils in the deposit. As an example, the distributions for $\gamma=0.4$ that present the minimum and maximum $\left|u_{z}\right|$ are shown in figure 5 and correspond to 13.4 and $19.0 \mathrm{~cm}$, respectively (i.e. a relative difference of $30 \%$ ). It is noted that for the minimum $\left|u_{z}\right|$ in figure 5a, the LMS elements (colored in blue) are mostly on shallow depths, under the structure and packed in groups or clusters. On 
the contrary, for the maximum $\left|u_{z}\right|$, there are smaller clusters and the LMS elements are surrounded by more denser sand in the mitigation zone. The different interactions between the LMS and MDS elements could be the cause of the difference in $\left|u_{z}\right|$. Thus, as more clusters develop (i.e. for very low or very high spatial fractions), more interactions develop between the two soils; therefore, more variation on the $\left|u_{z}\right|$ value is shown. In order to develop this explanation, three aspects will be further analyzed: i) the time history at one element under the structure right column, ii) the profile below the left column and iii) the entire model.

Firstly, the comparison of the time evolution of the liquefaction ratio $\left(r_{u}\right)$ between the homogeneous cases (i.e. before treatment -LMS- and fully treated -MDS) and the 20 simulations of heterogeneous cases is shown in figure 13 for one element. It is situated below the right column and at $2 \mathrm{~m}$ depth. Other positions were analyzed, but the response varies greatly with the confinement pressure and the effect of the structure. The comparison in the same element position of the different spatial distributions is interesting as it permits to analyze the numerous soil interactions (e.g. when the element is embedded in a cluster or in contact with the other soil) while leaving the mayority of other factors unchanged. Nonetheless, one could compare different positions in the same model but these will have different confinement pressures with distinct liquefaction evolution. For the sake of brevity, only one element is shown in the present analysis. The responses are divided in two figures: the behavior of the models where the element in the chosen position is composed of LMS are shown in green in figure 13a and those composed of MDS are shown in magenta in figure 13b.

Concerning figure 13a, it is interesting to note, that some LMS elements in the heterogeneous model present more pore pressure generation than those of the homogeneous LMS model. In contrast, some cases resemble the MDS model behavior. For these cases, 
the LMS element is principally surrounded by more denser sand, hence less pore-pressure migration can occur. In general, the behavior is affected by its neighbors but other LMS elements are also present.

Similarly in figure 13b, almost all MDS elements have higher $r_{u}$ than the corresponding homogeneous model. Additionally, for some MDS elements in the heterogeneous model the $r_{u}$ starts increasing after the predominant time of the motion (i.e. for $t>t_{95}=13 \mathrm{~s}$ ), while it decreases or stays constant for those composed of LMS, which emphasizes the interaction between the different soils. This interaction was also shown in the centrifuge analyses of Chakrabortty et al. (2010); although the comparison was made with a densityaverage homogeneous deposit and the elements compared were not at the same depth. While, in general, in the homogeneous deposits the pore-water pressure starts to dissipate at the end of shaking, the LMS elements in the heterogeneous model keep feeding water to neighboring MDS elements. This water migration has been analyzed by Chakrabortty et al. (1998), Ghosh and Madabhushi (2003), Popescu et al. (2005), among others.

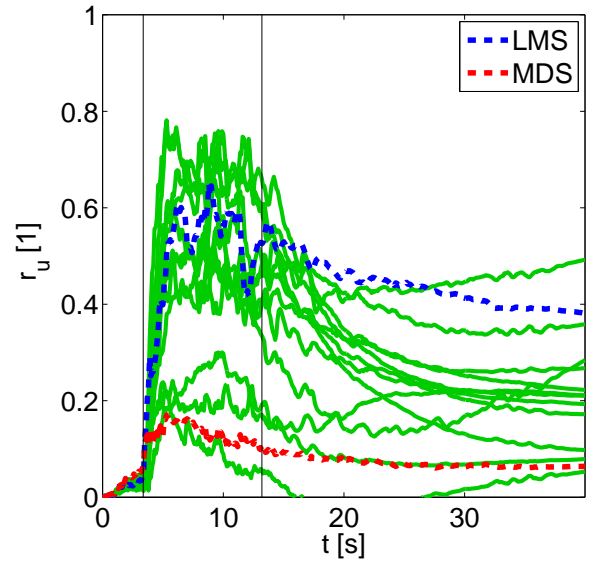

(a)

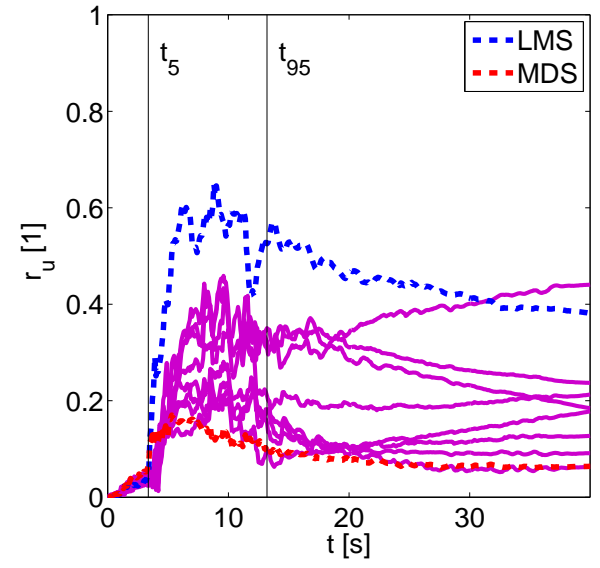

(b)

Figure 13.: Liquefaction ratio evolution with time for (a) LMS and (b) MDS elements at $2 \mathrm{~m}$ depth and under the right column for $\gamma=0.4$ and $\beta_{1}=\beta_{2}=0.4$. The homogeneous cases before (LMS) and after (MDS) treatment are also shown.

Two profiles below the left column of the structure are shown in figure 14 for all the 
spatial distributions used with $\gamma=0.4$ and $\beta_{1}=\beta_{2}=0.4$. The homogeneous models before (LMS) and after (MDS) treatment are also shown as reference. The $\gamma$ chosen presented the highest variation of the liquefaction index evaluated at the treated zone $\left(Q_{A}\right)$ shown in figure 10b. The profiles correspond to: a) the settlement and b) the Arias intensity $\left(I_{A}\right)$. Regarding the settlement profile in figure $14 \mathrm{a}$, it can be seen the important fluctuations in the heterogeneous zone and down to almost 10m. For the LMS model, the settlement is concentrated in the treated zone but for the MDS and most of the heterogeneous models, the settlement is greater for lower depths. This is reasonable because the MDS inclusions will stiffen the soil above, creating a higher impedance with the deeper zones where the profile will become more vulnerable.

In addition, figure $14 \mathrm{~b}$ shows the $I_{A}$ profile. This value is a measure of the total energy per unit mass defined by Arias (1970) as:

$$
I_{A}=\frac{\pi}{2} \int_{0}^{t_{\text {end }}} a(t)^{2} \mathrm{~d} t
$$

Its evolution expresses the amplification (or deamplification) of the energy through the soil profile. As $r_{u}$ increases and the soil softens, the seismic energy is deamplified by the soil. In contrast, as the heterogeneous models present less $r_{u}$ below $7 \mathrm{~m}$, the energy is amplified for deeper soil. However, as the $\gamma$ is small, the behavior of the heterogeneous model is closer to that of the MDS model. For all heterogeneous models, the $I_{A}$ at surface is higher than that of the untreated LMS model which will cause an amplification of the structure motion.

To compare the results of the entire deposit, figure 15 shows the differences between the heterogeneous model with maximum $\left|u_{z}\right|$ and the homogeneous LMS model. Again, results are shown for $\gamma=0.4$ and $\beta_{1}=\beta_{2}=0.4$, as an example. Figure 15a shows the $r_{u}$ differences (i.e. $r_{u H E T}-r_{u H O M}$ ) and it clearly depicts the interactions between the 


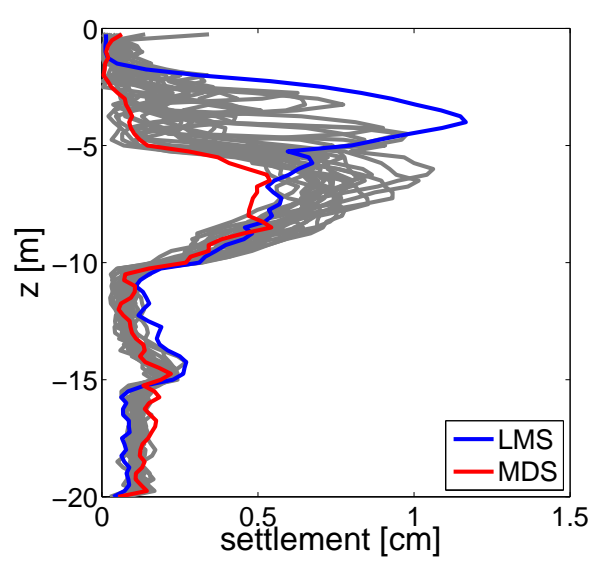

(a)

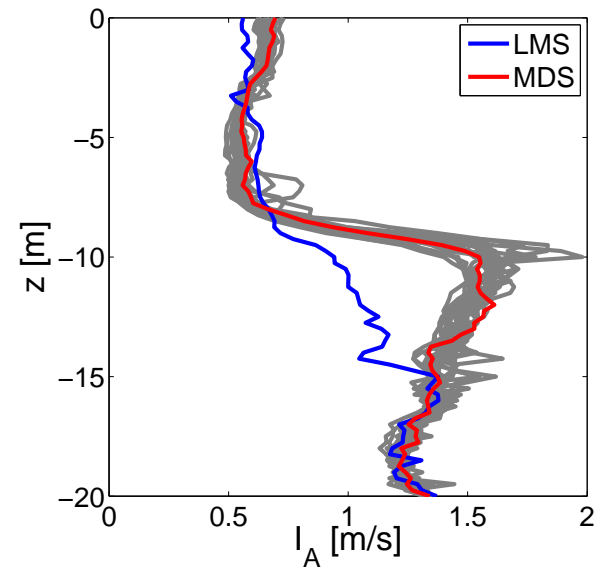

(b)

Figure 14.: Profile below the structure for (a) settlement and (b) Arias intensity at the end of shaking

two soils. As in the time histories (figure 13a), the results vary greatly from one position to another. For this case, the heterogeneous model presents, in general, lower $r_{u}$ values, specially in the treated zone (except for some regions). Although, immediately below the treated zone and at the bottom of the model, the $r_{u}$ increased after the inclusion of the denser sand. The explanation is that the interface around this zone acts as a barrier for the water migration and as the layered cases studied in the laboratory, a water film is formed around the liquefied sand (Fiegel and Kutter 1994, Kokusho and Kojima 2002, Konrad and Dubeau 2002, among others).

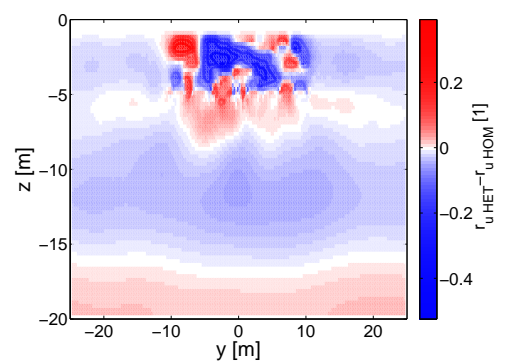

(a)

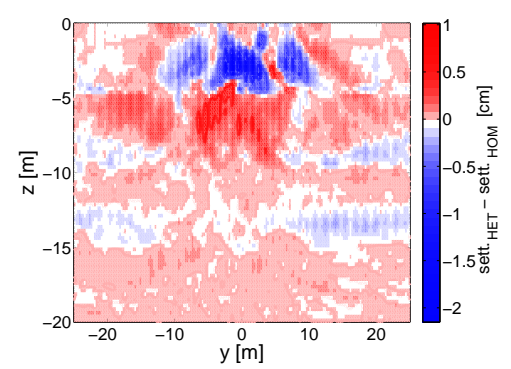

(b)

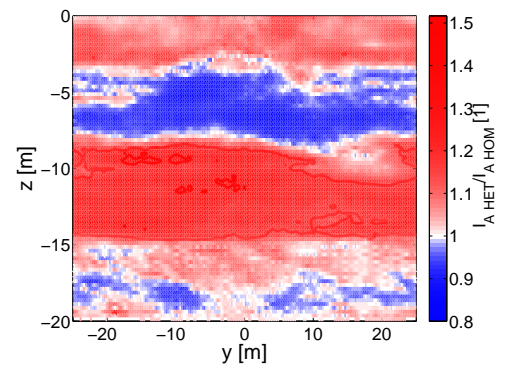

(c)

Figure 15.: Difference between the heterogeneous with maximum $\left|u_{z}\right|$ and the homogeneous LMS model for (a) the Liquefaction ratio $\left.\left(r_{u}\right), \mathrm{b}\right)$ the settlement and c) the Arias intensity $\left(I_{A}\right)$ at the end of shaking 
Similarly, the settlement at the end of shaking difference $($ sett.HET - settl.HOM $)$ between the heterogeneous and the homogeneous model before treatment is shown in figure 15b. As in the profile under the building column, before treatment the most part of the settlement is located below the structure, but after the heterogeneity is added, the highest settlement is found under the treated area. These two comparisons are evidence of the complexity added with the spatial variation by which neither the liquefaction apparition nor the settlement is homogeneous even for the untreated remaining soil, i.e. below and beside the intervened zone.

However, the comparison of the Arias intensity ratio (i.e. $I_{A H E T} / I_{A H O M}$ ), in figure $15 \mathrm{c}$, is mostly regular in the horizontal direction. The seismic motion is more amplified by the heterogeneous model for the soil near the surface and at the lower half of the soil deposit. The ratio is the lowest immediately below the treatment zone which corresponds to the highest values of liquefaction ratio in the heterogeneous model.

\section{Conclusions}

A numerical model of discrete spatial heterogeneity was used to assess the liquefaction induced settlement of an inelastic soil-structure system. Correlation in both directions was introduced by the nearest-neighbor model. The effect of the spatial fraction $(\gamma)$ of the treated-untreated soil mixture was analyzed. Twenty simulations were used for each of the $\gamma$. The main conclusions of the present study are:

- For the motion tested, the case of the fully-treated soil reduces the relative settlement of the structure with respect to free-field but increases the liquefaction ratio under and around the treated area.

- Owing to excess pore-pressure migration, treated soil surrounded by loose sand can present liquefaction and therefore low fractions of soil treatment have very low effi- 
ciency. Similarly, once the loose sand is well surrounded by treated soil, improvement in the relative settlement is small.

- While the relative settlement $\left|u_{z}\right|$ appears to be related to the liquefaction index $Q_{A}$; the latter presents more variation due to the pore-pressure migration. Even so, for some cases, $Q_{A}$ could be increased at adding denser soil to the deposit.

- In general, the spatial variability is highly dependent of the distribution of the two soils thus an average model will not be able to represent its behavior.

- Therefore, homogeneous equivalent models are to be taken only as reference and will rarely correspond to the arithmetic (i.e. parallel homogenization) nor harmonic (series homogenization) average of the heterogeneous response.

The numerical model of discrete spatial variation applied in a probabilistic framework appears to properly include heterogeneity on the soil. However, the effect of multivariate discrete models should be further analyzed. Furthermore, the effect of the soil properties correlation (in the horizontal and vertical direction) on the liquefaction-induced settlements should be investigated and, if possible, related to real in-situ measurements of added spatial variability due to mitigation techniques. The effect of different soil properties and different seismic motions could be also of interest.

\section{Acknowledgment}

The research reported in this paper has been supported in part by the SEISM Paris Saclay Research Institute and by the SINAPS@ project benefited from state funding managed by the National Research Agency under program RNSR Future Investments bearing reference No. ANR-11-RSNR- 0022-04. 


\section{References}

Alonso, E. and Krizek, R., 1975. Stochastic formulation of soil properties. In: 2nd Int. Conerence, Vol. 2 Aachen: Applications of Statistics and Probability in Soil and Structural Engineering, 9-32.

Arias, A., 1970. A mesure of earthquake intensity. In: Seismic Design of Nuclear Power Plants., 438-483 Cambridge,Massachusetts: MIT Press.

Aubry, D., et al., 1982. A double memory model with multiple mechanisms for cyclic soil behavior. In: International Symposium Num. Mod. Geomech. Balkema, 3-13.

Aubry, D. and Modaressi, A., 1996. GEFDyn - manuel scientifique. France: LMSSMat: Ecole Centrale Paris.

Bartlett, M. and Besag, J., 1969. Correlation Properties of Some Nearest-Neighbor Models. Bull. International Statistical Institute, 43 (2), 191-193.

Beresnev, I. and Wen, K., 1996. Nonlinear site response: a reality?. Bulletin of the Seismological Society of America, 86 (6), 1964-1978.

Bernardie, S., Foerster, E., and Modaressi, H., 2006. Non-linear site response simulations in Chang-Hwa region during the 1999 Chi-Chi earthquake, Taiwan. Soil dynamics and earthquake engineering, 26, 1038-1048.

Bird, J., et al., 2006. Modelling liquefaction-induced building damage in earthquake loss estimation. Soil Dynamics and Earthquake Engineering, 26, 15-30.

Bonilla, L., Gelis, C., and Regnier, J., 2011. The challenge of nonlinear site response: field data observations and numerical simulations. In: 4th IASPEI / IAEE International Symposium: Effects of Surface Geology on Seismic Motion, august University of Santa Barbara, p. 13.

Bradley, B.A., et al., 2013. Effect of lattice-shaped ground improvement geometry on 
seismic response of liquefiable soil deposits via 3-D seismic effective stress analysis. Soil Dynamics and Earthquake Engineering, 48 (1), 35-47.

Budiman, J.S., Mohammadi, J., and Bandi, S., 1995. Effect of Large Inclusions on Liquefaction of Sand. Geotech. Spec. Publ., 56 (1), 48-63.

Byrne, P., et al., 2004. Numerical modeling of liquefaction and comparison with centrifuge tests. Canadian Geotechnical Journal, 41 (2), 193-211.

Chakrabortty, P., Jafari-Mehrabadi, A., and Popescu, R., 1998. Effects of Low Permeability Soil Layers on Seismic Stability of Submarine Slopes. In: Proceedings of the 5\%th Canadian Geotechnical Conference, October Quebec,Canada: Canadian Geotechnical Society.

Chakrabortty, P. and Popescu, R., 2012. Numerical simulation of centrifuge tests on homogenous and heterogeneous soil models. Computers and Geotechnics, 41 (1), 95-105.

Chakrabortty, P., Popescu, R., and Phillips, R., 2010. Liquefaction of heterogeneous sand: Centrifuge study. Geotechnical testing journal, 33 (4), 1-11.

Coelho, P., et al., 2004. Centrifuge modeling of the use of densification as a liquefaction resistance measure for bridge foundations. In: 13th World Conference on Earthquake Engineering, Vol. 210, August Vancouver, B.C., Canada: WCEE.

Costa D'Aguiar, S., et al., 2011. Elastoplastic constitutive modelling of soil structure interfaces under monotonic and cyclic loading. Computers and Geotechnics, 38 (4), $430-447$.

Dashti, S., et al., 2010. Mechanisms of seismically-induced settlement of buildings with shallow foundations on liquefiable soil. J. Geotech. Geoenviron. Eng., 135 (1), 151164.

DeJong, J., et al., 2013. Bio-and chemo-mechanical processes in geotechnical engineering. 
Geotechnique, 63 (4), 287-301.

Fenton, G.A. and Vanmarcke, E.H., 1998. Spatial variation in liquefaction risk. Géotechnique, 48 (6), 819-831.

Fiegel, G. and Kutter, B., 1994. Liquefaction mechanism for layered soils. Journal of geotechnical engineering, 120 (4), 737-755.

Foerster, E. and Modaressi, H., 2007. Nonlinear numerical methods for earthquake site response analysis II- case studies. Bulletin of Earthquake Engineering, 5 (3), 325345.

Ghosh, B. and Madabhushi, S.P.G., 2003. Effects of Localized Soil Inhomogeneity in Modifying Seismic Soil-Structure Interaction. In: Proceedings of the 16th ASCE Engineering Mechanics Conference, July 16-18 Seattle,WA: American Society of Civil Engineers.

Giberson, M., 1969. Two nonlinear beams with definitions of ductility. Journal of structural division ASCE, 95 (2), 137-157.

Hausler, E. and Sitar, N., 2002. Performance of Improved Ground. In: Anual Meeting Research Digest, Vol. 16 University of California, Berkeley: Pacific Earthquake Engineering Research Center, p. 4.

Helton, J., et al., 2006. Survey of sampling-based methods for uncertainty and sensitivity analysis. Reliability engineering and system safety, 91 (10), 1175-1209.

Hujeux, J., 1985. Une loi de comportement pour le chargement cyclique des sols. In: Génie Parasismique., 278-302 v. davidovici ed. France: Presses ENPC.

Jones, A., Kramer, S., and Arduino, P., Estimation of Uncertainty in Geotechnical Properties for Performance-Based Earthquake Engineering. , 2002. , PEER Report 2002/16, Pacific Earthquake Engineering Research Center, University of California, Berkeley. 
Kasama, K., Whittle, A., and Zen, K., 2012. Effect of spatial variability on the bearing capacity of cement-treated ground. Soils and Foundations, 52 (4), 600-619.

Kokusho, T. and Kojima, T., 2002. Mechanism for postliquefaction water film generation in layered sand. Journal of Geotechnical and Geoenvironmental Engineering, 128 (2), $129-137$.

Konrad, J.M. and Dubeau, S., 2002. Cyclic strength of stratified soil samples. In: Proceedings of the 55th Canadian Geotechnical Conference: Ground and Water: Theory to Practice, October 20-25 Niagara Falls, Canada: Canadian Geotechnical Society, 89-94.

Koutsourelakis, S., Prévost, J., and Deodatis, G., 2002. Risk assessment of an interacting structure-soil system due to liquefaction. Earthquake Engineering and Structural Dynamics, 31, 851-879.

Lambert, S., Rocher-Lacoste, F., and Le Kouby, A., 2012. Soil-cement columns, an alternative soil improvement method. In: International Symposium on Ground Improvement Brussels, Belgium: ISSMGE - TC 211, p. 10.

Lopez-Caballero, F. and Modaressi-Farahmand-Razavi, A., 2013. Numerical simulation of mitigation of liquefaction seismic risk by preloading and its effects on the performance of structures. Soil dynamics and Earthquake engineering, 49 (1), 27-38.

Lopez-Caballero, F., Modaressi-Farahmand-Razavi, A., and Modaressi, H., 2007. Nonlinear numerical method for earthquake site response analysis I- elastoplastic cyclic model and parameter identification strategy. Bulletin of Earthquake Engineering, 5 (3), 303-323.

Maharjan, M. and Takahashi, A., 2012. Liquefaction Centrifuge Modeling in Nonhomogeneous Soil Deposits. In: 15th World Conference on Earthquake Engineering Lisboa, Portugal: WCEE. 
Mitchell, J., Cooke, H., and Schaeffer, J., 1998. Design considerations in ground improvement for seismic risk mitigation. In: Specialty Conf. on Geotechnical Earthquake Engineering and Soil Dynamics III, no. 75 in 1 Seattle,WA: American Society of Civil Engineers, 580-613.

Modaressi, H. and Benzenati, I., 1994. Paraxial approximation for poroelastic media. Soil Dynamics and Earthquake Engineering, 13 (2), 117-129.

Montoya-Noguera, S. and Lopez-Caballero, F., 2014. Effect of coupling excess pore pressure and deformation on nonlinear seismic soil response. Acta Geotechnica Online.

Paassen, L.V., 2011. Bio-Mediated Ground Improvement: From Laboratory Experiment to Pilot Applications. Geo-Frontiers 2011: Advances in Geotechnical Engineering, 4099-4108.

Popescu, R., Prevost, J.H., and Deodatis, G., 1998. Characteristic percentile of soil strength for dynamic analyses. Geotechnical earthquake engineering and soil dynamics, 1 (75), 1461-1471.

Popescu, R., Prevost, J.H., and Deodatis, G., 2005. 3D effects in seismic liquefaction of stochastically variable soil deposits. Géotechnique, 55 (1), 21-31.

Prakash, V., Powel, G., and Campbell, S., Chapter title. Base program description and user guide, DRAIN 2DX, 1993. .

Saez, E., 2009. Dynamic non-linear soil structure interaction. Thesis (PhD). Ecole Centrale Paris, France.

Saez, E., Lopez-Caballero, F., and Modaressi-Farahmand-Razavi, A., 2013. Inelastic dynamic soil-structure interaction effects on moment-resisting frame buildings. Engineering structures, 51 (1), 166-177.

Seed, H., et al., 1986. Moduli and damping factors for dynamic analyses of cohesionless soils. Journal of Geotechnical Engineering, 112 (11), 1016-1032. 
Senger, R., et al., 1992. Geostatistical/GeologicalPermeability Characterization of Carbonate Ramp Deposits in Sand Andres Outcrop , Algerita Escarpment, New Mexico. In: Permian Basin Oil and Gas Recovery Conference Midland, Texas: Society of Petroleum Engineers, 287-301.

Shinozuka, M. and Ohtomo, K., 1989. Spatial severity of liquefaction. In: Proceedings of the second US-Japan workshop in liquefaction, large ground deformation and their effects on lifelines., 193-206 NCEER.

Sica, S., Pagano, L., and Modaressi, A., 2008. Influence of past loading history on the seismic response of earth dams. Computers and Geotechnics, 35 (1), 61-85.

Vechio, F. and Emara, M., 1992. Shear deformation in reinforced concrete frames. ACI Structural journal, 89 (1), 46-56.

Whittle, P., 1963. Stochastic processes in several dimensions. Bulletin of the International Statistical Institute, 40, 974-994.

Wiener, O., 1912. Die theorie des Mischkorpers fur das feld des stationaren Stromung. Erste Abhandlung die Mittelswertsatze fur Kraft, Polarisation un Energie. Abhandlungen der mathematisch-physischen Klasse der Koniglich Sachsischen Gesellschaft der Wizzenschaften, 32 (6), 509-604.

Zienkiewicz, O. and Taylor, R., 1991. The Finite element method, solid and fluid mechanics, dynamics and non-linearity. 4th Vol. 2. London: McGraw-Hill Book Company. 